\title{
Editorial: The Role of Reactive Oxygen Species in Protective Immunity
}

\author{
Denis Martinvalet ${ }^{1,2 *}$ and Michael Walch ${ }^{3 *}$ \\ ${ }^{1}$ Department of Biomedical Sciences, University of Padua, Padova, Italy, ${ }^{2}$ Veneto Institute of Molecular Medicine, \\ Padova, Italy, ${ }^{3}$ Faculty of Science and Medicine, Department of Oncology, Microbiology and Immunology, Anatomy Unit, \\ University of Fribourg, Fribourg, Switzerland
}

Keywords: reactive oxygen species (ROS), protective immunity, innate immunity, adaptive immunity, immunoregulation

Editorial on the Research Topic

The Role of Reactive Oxygen Species in Protective Immunity

\section{INTRODUCTION}

Reactive oxygen species (ROS) result from the partial reduction of oxygen, and encompass both radical [superoxide anion $\left(\mathrm{O}_{2}{ }^{-}\right)$, hydroxyl radical $\left({ }^{\circ} \mathrm{OH}\right)$, and nitric oxide $(\mathrm{NO})$ ] and non-radical [hydrogen peroxide $\left(\mathrm{H}_{2} \mathrm{O}_{2}\right)$, hypochlorous acid $(\mathrm{HOCl})$, and peroxynitrite $\left(\mathrm{ONOO}^{-}\right)$] species having different half-life and reactivity. Classically, ROS have most often been considered harmful as they are common determinants of many cell death pathways including apoptosis, necrosis/necroptosis, ferroptosis, pyroptosis and autophagic cell death (1-8). This obvious ROS toxicity is certainly beneficial in oxidative killing of engulfed microbial pathogens in specialized immune cells, such as neutrophils (9) or macrophages (10). However, the simplistic view that ROS can only be harmful have been revisited. Indeed, ROS are essential to many physiological processes. ROS contribute to inflammation, vasoconstriction, signal transduction, cell migration, differentiation and proliferation (11-14). Moreover, $\mathrm{H}_{2} \mathrm{O}_{2}$ can modulate genes expression by redox based-epigenetic modification (15-17) and at the transcriptional level by activating redox responsive transcription factors such as AP-1, NRF2, CREB, HSF1, HIF-1, TP53, NF- $\mathrm{kB}$, NOTCH, SP1, SCREB-1 and FOXO family (18-26). $\mathrm{H}_{2} \mathrm{O}_{2}$ also acts at the posttranscriptional level to control gene expression by regulating both cap-dependent and cap-independent translation (27-29). Because of their rather pleiotropic actions, ROS are critically important for cell biology, organ function, and system physiology including that of the immune system. In this Research Topic, we aimed at taking a close look at how ROS contribute to protective immunity.

Received: 10 December 2021

Accepted: 21 December 2021

Published: 25 January 2022

Citation:

Martinvalet D and Walch M (2022) Editorial: The Role of Reactive Oxygen

Species in Protective Immunity.

Front. Immunol. 12:832946.

doi: 10.3389/fimmu.2021.832946

\section{ROS IN ANTIMICROBIAL IMMUNE DEFENSE}

ROS have been implicated in many aspects of the immune response to pathogens. ROS can damage biomolecules by oxidizing iron-sulfur clusters in a variety of enzymes leading to metabolic defects and release of iron. Free iron can react with hydrogen peroxide $\left(\mathrm{H}_{2} \mathrm{O}_{2}\right)$ to give rise to aggressive hydroxyl radicals that can damage any biomolecule, including DNA. Therefore, ROS are essential for pathogens 
killing by phagocytic cells as illustrated by chronic granulomatous disease (CGD), an inherited disorder of the NADPH oxidase characterized by recurrent and severe bacterial and fungal infections (30). Reciprocally, microbes developed many strategies to counteract ROS- dependent host defense mechanism as discuss by Sen and Imlay. Indeed, microbes sense the $\mathrm{H}_{2} \mathrm{O}_{2}$ via OxyR or PerR transcription factors, or use the Grx3/Yap1 system to initiate enzymes that reduce cytoplasmic $\mathrm{H}_{2} \mathrm{O}_{2}$ concentrations, decrease the intracellular iron pools, and repair the $\mathrm{H}_{2} \mathrm{O}_{2}$-mediated damage and suit their particular environmental niches.

In addition to the ability of ROS to directly kill bacteria in the phagosome by oxidative damage to essential biomolecules (31), they can also trigger pathogen defense of phagocytes by various nonoxidative means, such as autophagy, receptor signaling, extracellular traps formation and instructing lymphocyte responses. Interestingly, ROS critically orchestrate the inflammatory process by regulating cytokine production during infection. Adding to this line of research, Hatinguais et al. demonstrated that sublethal ROS were essential to proinflammatory cytokine expression in the context of macrophages infected with swollen Aspergillus fumigatus conidia. The source of these ROS was identified in mitochondria by reverse electron flow, although NADPH oxidase 2 seemed to play a regulatory role in this proinflammatory pathway. A study by Buvelot et al. showed that sublethal doses of hydrogen peroxide trigger a coordinated and massive downregulation of genes involved in pyrimidine metabolism in Staphylococcus aureus, leading to reduced growth of extracellular bacteria and an increased sensitivity to added $\mathrm{H}_{2} \mathrm{O}_{2}$. Strikingly, as opposed to extracellular bacteria, intracellular pathogens were less affected, which could be a long-term survival strategy by allowing colonization through intracellular survival, while decreasing the risk of killing the host through dampened extracellular growth.

The induction of caspase-mediated host cell death can critically contribute to the elimination of intracellular pathogens via the destruction of their niche and via the induction of efferocytosis (31-33). However, many obligate intracellular pathogens have evolved mechanisms to inhibit programed cells death. Lavergne et al. discuss how this limitation is dealt with by the cytotoxic lymphocyte proteases, the granzymes. In addition to triggering host cell death, they also exert various non-cytolytic antimicrobial activities by directly degrading vital microbial proteins or hijacked host proteins crucial for the replication or survival of the pathogens. The granzymes also target microbial virulent factors. Interestingly, many mechanisms applied by the granzymes in this context rely on the induction of reactive oxygen species, either by promoting host cell apoptosis or by inhibition of pathogen growth. Whether the ROS involved in pathogen killing, cell death induction and the regulation of host cell metabolism originated from the same source is not fully elucidated and will need further study.

\section{REGULATION OF IMMUNE CELLS BY ROS}

Beside the well-established ROS involvement in pathogen elimination, the wide range of the regulatory capacity of these reactive molecules is more and more revealed. Karmakar et al. discuss the regulatory mechanism downstream of the binding of Siglecs to sialic acid decorated receptor on immune cells. The Siglecs are a family of sialic-acid-binding immunoglobulin-like lectins believed to promote intercellular interactions and regulate the functions of innate and adaptive immune cells (34). Siglecs modulate immune activation and can promote or inhibit ROS generation under different contexts. dssSiglecs can bind sialoglycans present on the same cell (cis-interactions) or extracellular ligands present on neighboring cells or secretory glycoproteins (trans-interactions). Siglec-sialoglycan binding is weak and transient (22). The interaction between Siglecs with multivalent ligands leads to Siglec clustering, which increases the strength of Siglec-ligand binding and initiates cellular signaling.

ROS have also been proposed to be the common determinant of the inflammasome activation critical in the inflammatory process which is the determinant for an efficient immune response (35-38). The RNA-binding protein tristetraprolin (TTP) is an anti-inflammatory factor that prompts the mRNA decay of target mRNAs and is involved in inflammatory diseases such as rheumatoid arthritis (RA). lv et al. have shown that protein phosphatase 2A (PP2A)-mediated dephosphorylation regulates TTP to activate its $\mathrm{mRNA}$-degrading function. Interfering with TTP expression or agonist of PP2A modulate monosodium urate (MSU) crystal-induced the expression of inflammation-related genes and NLRP3 inflammasome activation in a mitochondrial ROS-dependent manner suggesting that targeting TTP expression or function may provide a potential therapeutic strategy for inflammation caused by MSU crystals.

These studies demonstrate that ROS exert highly pleotropic functions in immunity. Therefore, it is essential to identify the specific intracellular sources of ROS and how they influence cellular processes in both physiological and pathological means, and how they impact on metabolic processes and inflammatory signaling as discussed by Canton et al. in the context of macrophages. Moreover, ROS are also required for full activation of lymphocytes as well as for the regulation of autoimmunity. Indeed, Bassoy et al. highlight the contribution of ROS in lymphocyte biology and stress their contribution in adaptive immunity with direct impact on the outcome of the antitumoral immune response as a consequence of the redox state in the tumor microenvironment (TME). Cali et al. showed the detrimental tolerogenic role of tumor-infiltrating myeloid cells (TIMs) actively dismantling effective immunity against cancer. TIMs inhibit $\mathrm{T}$ cell functions and promote tumor progression by multiple mechanism including the potentiation of the oxidative/ nitrosative stress within the TME. They demonstrated that nitrosylation of granulocyte monocyte stimulating factor (GM$\mathrm{CSF}$ ) nourishes the expansion of this highly immunosuppressive myeloid subsets in tumor-bearing hosts.

The contribution of the ROS in adaptive immunity was further developed by Mortimer et al. who focus on the emerging role of NOX2-derived ROS in the development and maintenance of adaptive immunity and the effects of excess ROS in systemic disease. To this regard, Chávez and Tse discussed the 
impact of mitochondrial-derived ROS and immunometabolism reprogramming in autoreactive $\mathrm{T}$ cell differentiation. Dysfunctional mitochondria have been involved in oxidative stress associated with many $\mathrm{T}$ cell-mediated autoimmune diseases. This agrees with the ability of mitochondrial-derived ROS to also contributed to T cell fate and function. Therefore, targeted manipulation of glycolysis and mitochondrial derived ROS could contribute to the elimination of autoreactive T cells while promoting immunosuppressive CD4 T regulatory (Treg). This targeted manipulation would have the advantage of avoiding global immunosuppression and preserving physiological immune response.

Finally, given the essential role of ROS for basic physiological functions as well as their contribution to pathophysiological situation, their therapeutic manipulation becomes an attractive strategy, although a non-trivial one. Dumas and Knaus discuss essential consideration for effective Redox medicinal approaches.

\section{CONCLUSION}

This special Research Topic covers the extremely wide span of ROS function in immune defense and regulation and, with that, also the urgent need for further study to exploit these pleotropic

\section{REFERENCES}

1. Green DR, Kroemer G. The Pathophysiology of Mitochondrial Cell Death. Science (2004) 305:626-9. doi: 10.1126/science.1099320

2. Ricci JE, Munoz-Pinedo C, Fitzgerald P, Bailly-Maitre B, Perkins GA, Yadava $\mathrm{N}$, et al. Disruption of Mitochondrial Function During Apoptosis is Mediated by Caspase Cleavage of the P75 Subunit of Complex I of the Electron Transport Chain. Cell (2004) 117:773-86. doi: 10.1016/j.cell.2004.05.008

3. Cecconi F, Levine B. The Role of Autophagy in Mammalian Development: Cell Makeover Rather Than Cell Death. Dev Cell (2008) 15:344-57. doi: 10.1016/j.devcel.2008.08.012

4. Fulda S, Gorman AM, Hori O, Samali A. Cellular Stress Responses: Cell Survival and Cell Death. Int J Cell Biol (2010) 2010:214074. doi: 10.1155/2010/214074

5. Tait SW, Green DR. Mitochondria and Cell Death: Outer Membrane Permeabilization and Beyond. Nat Rev Mol Cell Biol (2010) 11:621-32. doi: $10.1038 / \mathrm{nrm} 2952$

6. Dixon SJ, Lemberg KM, Lamprecht MR, Skouta R, Zaitsev EM, Gleason CE, et al. Ferroptosis: An Iron-Dependent Form of Nonapoptotic Cell Death. Cell (2012) 149:1060-72. doi: 10.1016/j.cell.2012.03.042

7. Schenk B, Fulda S. Reactive Oxygen Species Regulate Smac Mimetic/ Tnfalpha-Induced Necroptotic Signaling and Cell Death. Oncogene (2015) 34:5796-806. doi: 10.1038/onc.2015.35

8. Fulda S. Regulation of Necroptosis Signaling and Cell Death by Reactive Oxygen Species. Biol Chem (2016) 397:657-60. doi: 10.1515/hsz-2016-0102

9. Nguyen GT, Green ER, Mecsas J. Neutrophils to the Roscue: Mechanisms of NADPH Oxidase Activation and Bacterial Resistance. Front Cell Infect Microbiol (2017) 7. doi: 10.3389/fcimb.2017.00373

10. Slauch JM. How Does the Oxidative Burst of Macrophages Kill Bacteria? Still an Open Question. Mol Microbiol (2011) 80:580-3. doi: 10.1111/j.13652958.2011.07612.x

11. Bedard K, Krause KH. The NOX Family of ROS-Generating NADPH Oxidases: Physiology and Pathophysiology. Physiol Rev (2007) 87:245-313. doi: 10.1152/physrev.00044.2005

12. Cash TP, Pan Y, Simon MC. Reactive Oxygen Species and Cellular Oxygen Sensing. Free Radic Biol Med (2007) 43:1219-25. doi: 10.1016/j.freeradbiomed.2007.07.001 molecules for therapy approaches. ROS are not only essential for antimicrobial defense but also exert a variety of crucial regulatory functions, reaching from the modulation of transcriptional programs to mediating differentiation fate. On the other hand, oxidative stress can contribute to various pathologies, including neuronal degenerative diseases, autoimmunity as well as cancer. Therefore, the potential therapeutic intervention in ROS biology will need extensive temporal and spatial fine tuning.

\section{AUTHOR CONTRIBUTIONS}

DM and MW wrote the manuscript. All authors contributed to the article and approved the submitted version.

\section{FUNDING}

Work from Martinvalet's lab was supported by grants from UNIPD SID 2018 and ERC starting grant ERC-2010StG_20091118. Work from the Walch's lab was supported by the Swiss National Science Foundation (SNSF grant \# 310030_169928), the Novartis Foundation for MedicalBiological Research and the Vontobel-Foundation (all to MW).
13. Winterbourn CC. Reconciling the Chemistry and Biology of Reactive Oxygen Species. Nat Chem Biol (2008) 4:278-86. doi: 10.1038/nchembio.85

14. Birben E, Sahiner UM, Sackesen C, Erzurum S, Kalayci O. Oxidative Stress and Antioxidant Defense. World Allergy Organ J (2012) 5:9-19. doi: 10.1097/ WOX.0b013e3182439613

15. Bosch-Presegue L, Raurell-Vila H, Marazuela-Duque A, Kane-Goldsmith N, Valle A, Oliver J, et al. Stabilization of Suv39H1 by Sirt1 Is Part of Oxidative Stress Response and Ensures Genome Protection. Mol Cell (2011) 42:210-23. doi: 10.1016/j.molcel.2011.02.034

16. Cyr AR, Domann FE. The Redox Basis of Epigenetic Modifications: From Mechanisms to Functional Consequences. Antioxid Redox Signal (2011) 15:551-89. doi: 10.1089/ars.2010.3492

17. Kreuz S, Fischle W. Oxidative Stress Signaling to Chromatin in Health and Disease. Epigenomics (2016) 8:843-62. doi: 10.2217/epi-2016-0002

18. Schreck R, Rieber P, Baeuerle PA. Reactive Oxygen Intermediates as Apparently Widely Used Messengers in the Activation of the NF-Kappa B Transcription Factor and HIV-1. EMBO J (1991) 10:2247-58. doi: 10.1002/ j.1460-2075.1991.tb07761.x

19. Allen RG, Tresini M. Oxidative Stress and Gene Regulation. Free Radic Biol Med (2000) 28:463-99. doi: 10.1016/S0891-5849(99)00242-7

20. Finkel T, Holbrook NJ. Oxidants, Oxidative Stress and the Biology of Ageing. Nature (2000) 408:239-47. doi: 10.1038/35041687

21. Bonello S, Zahringer C, Belaiba RS, Djordjevic T, Hess J, Michiels C, et al. Reactive Oxygen Species Activate the HIF-1alpha Promoter via a Functional Nfkappab Site. Arterioscler Thromb Vasc Biol (2007) 27:755-61. doi: 10.1161/ 01.ATV.0000258979.92828.bc

22. Matsuzawa A, Ichijo H. Redox Control of Cell Fate by MAP Kinase: Physiological Roles of ASK1-MAP Kinase Pathway in Stress Signaling. Biochim Biophys Acta (2008) 1780:1325-36. doi: 10.1016/j.bbagen.2007.12.011

23. Akasaki Y, Alvarez-Garcia O, Saito M, Carames B, Iwamoto Y, Lotz MK. Foxo Transcription Factors Support Oxidative Stress Resistance in Human Chondrocytes. Arthritis Rheumatol (2014) 66:3349-58. doi: 10.1002/art.38868

24. Marinho HS, Real C, Cyrne L, Soares H, Antunes F. Hydrogen Peroxide Sensing, Signaling and Regulation of Transcription Factors. Redox Biol (2014) 2:535-62. doi: 10.1016/j.redox.2014.02.006 
25. Espinosa-Diez C, Miguel V, Mennerich D, Kietzmann T, Sanchez-Perez P, Cadenas S, et al. Antioxidant Responses and Cellular Adjustments to Oxidative Stress. Redox Biol (2015) 6:183-97. doi: 10.1016/j.redox. 2015.07.008

26. Weidinger A, Kozlov AV. Biological Activities of Reactive Oxygen and Nitrogen Species: Oxidative Stress Versus Signal Transduction. Biomolecules (2015) 5:472-84. doi: 10.3390/biom5020472

27. Stoneley M, Willis AE. Cellular Internal Ribosome Entry Segments: Structures, Trans-Acting Factors and Regulation of Gene Expression. Oncogene (2004) 23:3200-7. doi: 10.1038/sj.onc.1207551

28. Li W, Thakor N, Xu EY, Huang Y, Chen C, Yu R, et al. An Internal Ribosomal Entry Site Mediates Redox-Sensitive Translation of Nrf2. Nucleic Acids Res (2010) 38:778-88. doi: 10.1093/nar/gkp1048

29. Zhang J, Dinh TN, Kappeler K, Tsaprailis G, Chen QM. La Autoantigen Mediates Oxidant Induced De Novo Nrf2 Protein Translation. Mol Cell Proteomics (2012) 11:M111 015032. doi: 10.1074/mcp.M111.015032

30. O'neill S, Brault J, Stasia MJ, Knaus UG. Genetic Disorders Coupled to ROS Deficiency. Redox Biol (2015) 6:135-56. doi: 10.1016/j.redox.2015.07.009

31. Dupre-Crochet S, Erard M, Nuss O. ROS Production in Phagocytes: Why, When, and Where? J Leukocyte Biol (2013) 94:657-70. doi: 10.1189/jlb.1012544

32. Labbe K, Saleh M. Cell Death in the Host Response to Infection. Cell Death Differ (2008) 15:1339-49. doi: 10.1038/cdd.2008.91

33. Behar SM, Briken V. Apoptosis Inhibition by Intracellular Bacteria and Its Consequence on Host Immunity. Curr Opin Immunol (2019) 60:103-10. doi: 10.1016/j.coi.2019.05.007

34. Crocker PR, Paulson JC, Varki A. Siglecs and Their Roles in the Immune System. Nat Rev Immunol (2007) 7:255-66. doi: 10.1038/nri2056
35. Dostert C, Petrilli V, Van Bruggen R, Steele C, Mossman BT, Tschopp J. Innate Immune Activation Through Nalp3 Inflammasome Sensing of Asbestos and Silica. Science (2008) 320:674-7. doi: 10.1126/science.1156995

36. Schroder K, Tschopp J. The Inflammasomes. Cell (2010) 140:821-32. doi: 10.1016/j.cell.2010.01.040

37. Zhou R, Tardivel A, Thorens B, Choi I, Tschopp J. Thioredoxin-Interacting Protein Links Oxidative Stress to Inflammasome Activation. Nat Immunol (2010) 11:136-40. doi: 10.1038/ni.1831

38. Zhou R, Yazdi AS, Menu P, Tschopp J. A Role for Mitochondria in NLRP3 Inflammasome Activation. Nature (2011) 469:221-5. doi: 10.1038/nature09663

Conflict of Interest: The authors declare that the research was conducted in the absence of any commercial or financial relationships that could be construed as a potential conflict of interest.

Publisher's Note: All claims expressed in this article are solely those of the authors and do not necessarily represent those of their affiliated organizations, or those of the publisher, the editors and the reviewers. Any product that may be evaluated in this article, or claim that may be made by its manufacturer, is not guaranteed or endorsed by the publisher.

Copyright $\odot 2022$ Martinvalet and Walch. This is an open-access article distributed under the terms of the Creative Commons Attribution License (CC BY). The use, distribution or reproduction in other forums is permitted, provided the original author(s) and the copyright owner(s) are credited and that the original publication in this journal is cited, in accordance with accepted academic practice. No use, distribution or reproduction is permitted which does not comply with these terms. 